\title{
Prognostic Factors of Complications After Thoracoscopic Lobectomy for Non-Small Cell Lung Cancer-A Retrospective Study
}

\section{Kai Wang}

the First Affiliated Hospital of Nanjing Medical University

\section{Derun Xia}

Nanjing Medical University

Yi Yin

Nanjing Medical University

\section{Yu Wang}

the First Affiliated Hospital of Nanjing Medical University

\section{Sibo Sun}

the First Affiliated Hospital of Nanjing Medical University

\section{Mingjiong Zhang}

the First Affiliated Hospital of Nanjing Medical University

\section{Wen liu}

the First Affiliated Hospital of Nanjing Medical University

\section{Shuangshuang Wu}

the First Affiliated Hospital of Nanjing Medical University

zhihang Peng

Nanjing Medical University

Jianqing Wu ( $\nabla$ jwunjmu@163.com )

the First Affiliated Hospital of Nanjing Medical University

\section{Research Article}

Keywords: video-assisted thoracoscopic surgery, non-small cell lung cancer, postoperative cardiopulmonary complications

Posted Date: May 28th, 2021

DOl: https://doi.org/10.21203/rs.3.rs-515147/v1

License: (c) (1) This work is licensed under a Creative Commons Attribution 4.0 International License.

Read Full License 


\section{Abstract}

Background: Video-assisted thoracoscopic surgery, safe and minimally invasive, is the first strategy recommended for non-small cell lung cancer. The purpose of this study was to determine the risk factors for postoperative cardiopulmonary complications (including cardiac and pulmonary complications) in patients with NSCLC who underwent video-assisted thoracoscopic surgery (VATS).

Methods: We retrospectively collected information of 3142 lung cancer patients undergoing VATS tumor resection at Jiangsu Provincial People's Hospital from January 2017 to June 2018, and established a clinical prediction model using the factors selected by univariate analysis.

Results: A total of 305 in 3142 patients developed postoperative cardiopulmonary complications. In univariate analysis, age, $\mathrm{PNI}, \mathrm{CCl}$, long-term smoking history before surgery, conversion to thoracotomy, albumin before surgery, pre-albumin, $\Delta$ albumin and pleura adhesion were all associated with cardiac and pulmonary complications. Multivariate analysis showed that age, $\mathrm{PNI}, \mathrm{CCl}$, long-term smoking history before surgery, conversion to thoracotomy, pre-albumin, $\Delta$ albumin were important independent risk factors for complications. Finally, age, $\mathrm{PNI}, \mathrm{CCl}$, long-term smoking history before surgery, conversion to thoracotomy, $\triangle$ albumin variables were included in the model (AUC=0.743).

Conclusion: Nutritional status (PNI, pre-albumin), CCl, age, long-term smoking history before surgery, conversion to thoracotomy were all independent risk factors for postoperative complications and poor prognosis. Experienced surgeons should instruct the operation of high-risk patients to avoid long-term hospitalization and possible poor prognosis after surgery.

\section{Introduction}

According to the latest global cancer burden data released by the International Agency for Cancer Research (IARC) of the World Health Organization in 2020, lung cancer is still one of the diseases with the highest morbidity and mortality in the world[1]. For early stage lung cancer, video-assisted thoracoscopic surgery (VATS) is still the main treatment strategy[2].

Ever since its invention, VATS has gradually become the main treatment for non-small cell lung cancer (NSCLC)[3]. However, postoperative cardiopulmonary complications (PCCs) still plague surgeons. According to our study cohort, the overall incidence of cardiopulmonary complications after thoracoscopy was $24.9-36.5 \%$ [4-6]. In related studies, age, nutritional status, CCI, long-term smoking history before surgery (LSHS) and conversion to thoracotomy were proven as risk factors for PCCs. PCCs leads to longer hospital stay and heavier economic burden for medical care [7]. Therefore, predicting and preventing PCCs in patients before surgery are of positive significance.

According to previous studies, albumin (ALB) showed certain predictive significance for postoperative complications of digestive system tumors, such as oral cancer, major abdominal surgery, colorectal cancer, and gastric cancer [8-11]. Serum ALB as an important factor to preoperative risk stratification 
has been used in the prediction of postoperative outcomes [12]. In a retrospective study conducted by West China Hospital in China, perioperative serum ALB level was used as an independent predictor to predict the occurrence of PCCs. However, Loftus held the opinion that serum ALB could only reflect nutritional status when the visceral protein is stable [12]. Therefore, some studies have proposed to use PNI to predict postoperative lung cancer risk [13], and found that the incidence of pneumothorax, pneumonia and extrapulmonary infection in the PNI group was significantly higher than that in the normal PNI group.

In addition to nutritional status, age and CCI may also be predictors of PCCs. Some studies found that elderly patients were more likely to have PCCs $[14,15]$, but a prospective American research suggested that age could not predict the occurrence of major postoperative complications [16], and one patient's physique-related indexes could better predict the occurrence of PCCs. The Charlson Comorbidity Index $(\mathrm{CCl})$ is also a good indicator for evaluating postoperative complications $[15,17]$, and $\mathrm{CCl}$ has many overlaps with the criteria for assessing frailty, so both can reflect frailty. In addition, it is still controversial whether intraoperative conversion to thoracotomy and LSHS before surgery can be used as risk factors for PCCs. Two retrospective studies suggested that the different survivals of patients who received successful VATS and converted to thoracotomy. There was no significant difference in recurrence $[18,19]$, but the incidences of atrial fibrillation, pneumothorax, and acute renal failure were higher in patients who converted to thoracotomy in Bongiolatti S' study [20].

Studies showed that patients with LSHS in forefoot surgery were more likely to develop PCCs[21, 22]. However, in the studies of spinal surgery, head and neck surgery, there was no correlation between LSHS and complications $[23,24]$. There are also different voices on whether the clinical stage of lung cancer can predict the incidence of PCCs $[25,26]$

In this study, we retrospectively collected the incidences of PCCs in 3142 patients who underwent VATS for NSCLC in Jiangsu Provincial People's Hospital, and evaluated various factors related to PCCs, including age, gender, PNI, CCI, LSHS and $\triangle \mathrm{ALB}$. We established a prediction model based on the data of all patients in order to evaluate the incidence of PCCs before surgery.

\section{Materials And Methods}

\section{Study patients}

Totally, 3149 cases recruited from Jiangsu People Hospital were divided into groups A and B. After data processing, we deleted samples with $>25 \%$ dada missed and meaningless data. The final sample size was 3142 , consisting of 305 patients who had PCCs and 2837 patients who had never developed any complications. Inclusion criteria were: (1) target diseases were operable primary NSCLCs; (2) only standardized single-lobectomy with systematic mediastinal lymph node dissection (SMLND) was operated by a VATS procedure; (3) patients must finish the entire clinical pathway according to our institutional policies during the hospitalization; (4) patients with loss of accurate records on estimated variables were not considered. 


\section{Data collection}

Baseline data collected for this study included: age, gender, BMI, LSHS, CCI (Charlson Comorbidity Index), operation time, FEV1, TNM staging, conversion to thoracotomy, WBC, ALB before operation, albumin change $(\triangle \mathrm{ALB}), \mathrm{LDL}$, postoperative stage, tumor location and pleura adhesion. Among these variables, the pleura adhesion was classified into four types (no, light, medium, and high); tumor location into five types (the top right, top left, bottom right, bottom left and middle right group); smoking history into two types (non-smoking and more than 10 years). $\mathrm{CCl}$ is the Chalson comorbidity index [17]. Constantly, blood samples were measured at 1-7 days before surgery and one day after surgery.

\section{Statistical analysis}

After data processing, we described continuous normally distributed data with mean \pm standard deviation (SD) and non-normal data with medians and corresponding interquartile range (IQR) (25\% and 75\% percentiles). Categorical variables were expressed as absolute frequencies and proportions (\%). Clinicopathologic characteristics were compared between the two groups. For categorical variable, chisquare method was used to analyze the difference between two groups. For continuous variable, student t-test and variance analysis were used for variables with normality and variance homogeneity, while Wilcoxon rank sum test for variables failed to satisfying the requirements above. Variables with significant meaning $(P<0.05)$ were selected for extra analysis, and the relative results of difference analysis were visualized. Then we established multivariable logistics regression model using the variables with significant difference defined in univariate analysis. Results of regression analysis were expressed as odds ratio (ORs) and 95\% confidence interval (95\%). The receiver operating characteristics (ROC) curve was drawn for evaluating the model, and nomogram analysis was performed to describe the variable scores for risk prediction. All statistics analysis was performed in R software 4.0.2 version.

\section{Results}

A total of 3142 patients undergoing VATS were included in this retrospective study. Table 1 summarizes the baseline characteristics. The average age was $56(48,64)$ years, $57.7 \%(1813 / 4142)$ of the patients were women, and the average preoperative ALB level was $43.6(40.5,47.8) \mathrm{g} / \mathrm{L}$. Hypoalbuminemia was defined in 235 patients with preoperative albumin lower than $35 \mathrm{~g} / \mathrm{L}$. The average albumin decreased to $37.7(34.8,40.3) \mathrm{g} / \mathrm{L}$ on the first day after surgery. The numbers of patients with $\mathrm{CCl}$ grades 0,1 , >=2 were 1825 (58\%), 898 (28.6\%), 421 (13.4\%), respectively. During the operation, 82 patients converted to thoracotomy for various reasons. The average preoperative WBC level was $8.51 \mathrm{~g} / \mathrm{L}[6.2,11.9]$, and the average LDL level was $2.62[2.1,3.1] \mathrm{g} / \mathrm{L}$. Preoperative TNM stages 1,2 and 3 were observed in 2962, 124 and 63 patients, respectively. $54.7 \%(, p<0.001)$ of patients had different degrees of pleural adhesions. 
Table 1

Baseline charcateristics of patients $(n=3142)$ who had undergone VATS

\begin{tabular}{|c|c|c|c|c|}
\hline & Overall & $\begin{array}{l}\text { No } \\
\text { Complication } \\
\text { (\%) }\end{array}$ & $\begin{array}{l}\text { Complication } \\
\text { (\%) }\end{array}$ & $\mathbf{p}$ \\
\hline$n$ & 3142 & 2837 & 305 & \\
\hline age (median [IQR]) & $56[48,65]$ & $56[48,64]$ & $62[54,67]$ & $<001$ \\
\hline gender = Female $(\%)$ & $1813(57.7)$ & $1643(57.9)$ & $170(55.7)$ & 0.4649 \\
\hline PNI (median [IQR]) & $\begin{array}{l}43.60[40.55 \\
47.80]\end{array}$ & $\begin{array}{l}43.85[40.75 \\
48.10]\end{array}$ & $\begin{array}{l}41.30[38.75 \\
44.20]\end{array}$ & $\stackrel{<}{0.001}$ \\
\hline CCI (\%) & & & & $<.001$ \\
\hline 0 & $1823(58.0)$ & $1684(59.4)$ & $139(45.6)$ & \\
\hline 1 & $898(28.6)$ & $804(28.3)$ & $94(30.8)$ & \\
\hline$>=2$ & $421(13.4)$ & 349 (12.3) & $72(23.6)$ & \\
\hline BMI (median [IQR]) & $\begin{array}{l}22.00[20.22, \\
23.90]\end{array}$ & $\begin{array}{l}22.00[20.20 \\
23.90]\end{array}$ & $\begin{array}{l}22.00[20.30 \\
24.10]\end{array}$ & 0.413 \\
\hline Smoking history (\%) & $1208(38.4)$ & $1056(37.2)$ & $152(49.8)$ & $\begin{array}{l}< \\
0.001\end{array}$ \\
\hline operation.time $\geq 2 \mathrm{~h}(\%)$ & $603(19.2)$ & $541(19.1)$ & $62(20.3)$ & 0.5959 \\
\hline $\mathrm{FEV} 1=<60 \%(\%)$ & $492(15.7)$ & $437(15.4)$ & $55(18.0)$ & 0.2299 \\
\hline $\begin{array}{l}\text { conversion.to.thoracotomy = } \\
\text { Thoracotomy (\%) }\end{array}$ & $82(2.6)$ & $60(2.1)$ & $22(7.2)$ & $<001$ \\
\hline WBC (median [IQR]) & $\begin{array}{l}8.51[6.20 \\
11.94]\end{array}$ & $\begin{array}{l}8.12[6.00 \\
11.40]\end{array}$ & $\begin{array}{l}11.83 \text { [10.10, } \\
14.09]\end{array}$ & $\begin{array}{l}< \\
0.001\end{array}$ \\
\hline albumin.b (median [IQR]) & $\begin{array}{l}42.80[39.00 \\
48.00]\end{array}$ & $\begin{array}{l}42.80[38.90 \\
47.80]\end{array}$ & $\begin{array}{l}43.00[39.60 \\
50.40]\end{array}$ & 0.024 \\
\hline albumin.change (median [IQR]) & $\begin{array}{l}4.90[2.20, \\
8.40]\end{array}$ & $\begin{array}{l}4.70[2.10 \\
7.90]\end{array}$ & $\begin{array}{l}6.90[3.80 \\
14.60]\end{array}$ & $\begin{array}{l}< \\
0.001\end{array}$ \\
\hline LDL (median [IQR]) & $\begin{array}{l}2.62[2.15, \\
3.14]\end{array}$ & $\begin{array}{l}2.64[2.16, \\
3.17]\end{array}$ & $\begin{array}{l}2.49[2.01 \\
2.92]\end{array}$ & $\begin{array}{l}< \\
0.001\end{array}$ \\
\hline TNM & & & & 0.166 \\
\hline ૧ & 2955(94) & 2662(93.8) & 293(96.0) & \\
\hline Q & 124(3.9) & 114(4) & 10(3.2) & \\
\hline Q & $63(2)$ & $61(2.1)$ & $2(0.6)$ & \\
\hline
\end{tabular}




\begin{tabular}{|lllll|}
\hline & Overall & $\begin{array}{l}\text { No } \\
\text { Complication } \\
(\%)\end{array}$ & $\begin{array}{l}\text { Complication } \\
(\%)\end{array}$ & p \\
\hline ASA score & & & & 0.950 \\
\hline 2 & $2607(82.9)$ & $2352(82.9)$ & $255(83.6)$ & \\
\hline 3 & $441(14)$ & $400(14)$ & $41(13.4)$ & \\
\hline 4 & $14(2.9)$ & $85(2.9)$ & $9(2.9)$ & 0.329 \\
\hline tumor.location (\%) & & & & \\
\hline Top.right & $969(30.8)$ & $884(31.2)$ & $85(27.9)$ & \\
\hline Top.left & $406(12.9)$ & $363(12.8)$ & $43(14.1)$ & \\
\hline Bottom.right & $786(25.0)$ & $718(25.3)$ & $68(22.3)$ & \\
\hline Bottom.left & $507(16.1)$ & $452(15.9)$ & $55(18.0)$ & \\
\hline Middle.right & $474(15.1)$ & $420(14.8)$ & $54(17.7)$ & \\
\hline Pleural adhesion (\%) & & & $155(50.8)$ & \\
\hline No & $1422(45.3)$ & $1267(44.7)$ & $62(26.9)$ & \\
\hline Light & $1011(32.2)$ & $929(32.7)$ & $62(20.3)$ & \\
\hline Medium & $586(18.7)$ & $524(18.5)$ & $117(4.1)$ & $6(2.0)$ \\
\hline High & $123(3.9)$ & & & \\
\hline
\end{tabular}

[Surgery results]: A total of 305 people had experienced at least one PCC. The total incidence of PCCs was $7.4 \%$ (305/4142), and no patient died in the hospital. Among all complications, pneumothorax accounted for the largest proportion $(3.1 \%, 127 / 4142)$.

[Comparison between PCCs group and non-PCCs group]: Table 1 shows the perioperative demographic difference between patients with or without PCCs. Figure Table1 respectively show the distribution and differences of age, PNI, SIRI, $\triangle A L B, C C I$, LSHS between the two groups with or without PCCs. Age ( $p<$ $0.001)$, WBC $(p<0.001)$ and ALB $(P=0.024)$ were higher in the PCCS group, while PNI $(P<0.001)$ was lower in the PCCs group, and the median differences in these variables between the two groups were statistically significant. Meanwhile, $\mathrm{CCl}(\mathrm{p}<0.001)$, conversion to thoracotomy $(p<0.001)$ showed difference between the two groups.

[Intraoperative variables]: In terms of intraoperative parameters. Conversion to thoracotomy during operation $(p<0.001)$, ratio of $\triangle A L B(p<0.001)$ and pleural adhesions $(p=0.029)$ in PCCs group was higher than those in non-PCC patients. $\triangle$ ALB of patients with PCCs was $6.9(3.8,14.6) \mathrm{g} / \mathrm{L}$, while that of patients without PCCs was $4.7(2.1,7.9) \mathrm{g} / \mathrm{L}$. 
[Pathological results]: Patients with PCCs had significantly higher incidence of tumor invasion $(p=0.002)$. The proportions of patients in TNM stages 2 and $3(p=0.027)$ and other pathological parameters showed no significant difference in patients without PCC.

[Univariate and multivariate analyses]: As shown in Table 2, our multivariate binary regression model included 16 variables related to PCC risk. Finally, we found that age $(1.04(1.03,1.05), p<0.001)$, LSHS $(1.68(1.32,2.12), p<0.001)$, intraoperative conversion to thoracotomy $(3.60(2.13,5.87), p<0.001), \triangle A L B$ value $(1.05(1.04,1.07), p<0.001)$ were independent risk factors for PCCs under VATS. Among the multicategorical variables, $\mathrm{CCl}(2.39(1.72,3.31), \mathrm{p}<0.001)$, PNI $(0.38(0.20,0.72, \mathrm{p}=0.004))$ was considered as an independent risk factors for PCCs under VATS. With PNI $<45$ as the baseline, the risk of PNI $>50$ was 0.38 times higher $(0.2,0.72, p=0.002)$. In addition, $\operatorname{LDL}(0.71(0.60,0.84) p<0.001)$ and pleural adhesions $(0.42(0.16,0.89) p=0.04)$ were only statistically significant in univariate analysis, but after other parameters were included, the adjusted OR values were all greater than 0.05 . 
Table 2

Relationships between postoperative complications of VATS and clinicopathological characteristics

\begin{tabular}{|c|c|c|c|c|}
\hline & $\begin{array}{l}\text { Unadjusted OR } \\
(95 \% \mathrm{Cl})\end{array}$ & P-value & $\begin{array}{l}\text { Adjusted OR } \\
(95 \% \mathrm{Cl})\end{array}$ & P-value \\
\hline Age & $1.04(1.03-1.05)$ & $<0.001$ & $1.03(1.02-1.04)$ & $<0.001$ \\
\hline Gender(female) & $0.92(0.72-1.16)$ & 0.47 & - & - \\
\hline BMI & $1.02(0.98-1.06)$ & 0.42 & - & - \\
\hline LSHS & $1.68(1.32-2.12)$ & $<0.001$ & $1.64(1.27-2.10)$ & $<0.001$ \\
\hline \multicolumn{5}{|l|}{$\mathrm{CCl}$} \\
\hline 0 & - & - & - & - \\
\hline 1 & $1.42(1.07-1.86)$ & 0.01 & $1.35(1.01-1.79)$ & 0.04 \\
\hline$>=2$ & $2.50(1.83-3.39)$ & $<0.001$ & $2.39(1.72-3.31)$ & $<0.001$ \\
\hline PNI & $0.90(0.88-0.92)$ & $<0.001$ & & \\
\hline$<45$ & - & - & - & - \\
\hline $45-50$ & $0.36(0.25-0.50)$ & $<0.001$ & $0.52(0.34-0.77)$ & 0.002 \\
\hline$>=50$ & $0.24(0.14-0.39)$ & $<0.001$ & $0.38(0.20-0.72)$ & 0.004 \\
\hline Operation time $(>2 \mathrm{~h})$ & $1.08(0.80-1.44)$ & 0.60 & - & - \\
\hline FEV1 $(<60 \%)$ & $1.21(0.88-1.63)$ & 0.23 & - & - \\
\hline ASA & & 0.950 & & \\
\hline 2 & - & - & - & - \\
\hline 3 & $1.02(0.51-2.1)$ & 0.947 & & \\
\hline 4 & $0.97(0.45-2.07)$ & 0.933 & & \\
\hline Conversion to thoracotomy & $3.60(2.13-5.87)$ & $<0.001$ & $3.54(2.03-5.99)$ & $<0.001$ \\
\hline WBC & $1.00(1.00-1.00)$ & 0.32 & - & - \\
\hline Pre-ALB & $1.01(1.00-1.03)$ & 0.04 & $0.94(0.90-0.98)$ & 0.004 \\
\hline$\triangle \mathrm{ALB}$ & $1.05(1.04-1.07)$ & $<0.001$ & $1.14(1.08-1.19)$ & $<0.001$ \\
\hline LDL & $0.71(0.60-0.84)$ & $<0.001$ & - & - \\
\hline Postoperative.stage & & 0.198 & & \\
\hline Q & - & - & - & - \\
\hline
\end{tabular}




\begin{tabular}{|lllll|}
\hline & $\begin{array}{l}\text { Unadjusted OR } \\
(95 \% \mathrm{Cl})\end{array}$ & P-value & $\begin{array}{l}\text { Adjusted OR } \\
(95 \% \mathrm{Cl})\end{array}$ & P-value \\
\hline$\square$ & $3.36(0.82,13.80)$ & 0.093 & \\
\hline Tumor.location & $2.68(0.57,12.60)$ & 0.213 & & \\
\hline Top.right & & & & - \\
\hline Top.left & - & - & - & - \\
\hline Bottom.right & $1.23(0.83-1.80)$ & 0.29 & - & - \\
\hline Bottom.left & $0.98(0.70-1.37)$ & 0.93 & - & - \\
\hline Middle.right & $1.27(0.88-1.80)$ & 0.2 & - & - \\
\hline Pleura.adhesion & $1.34(0.93-1.91)$ & 0.11 & - & - \\
\hline No & & & & - \\
\hline Light & - & - & - & - \\
\hline Medium & $0.72(0.54-0.95)$ & 0.02 & - & - \\
\hline High & $0.97(0.70-1.31)$ & 0.83 & - & - \\
\hline
\end{tabular}

[Nomatograph and model prediction]: Finally, we evaluated the significances of age, CCl, PNI, LSHS, conversion to thoracotomy and $\triangle A L B$ based on the results of univariate and multivariate analyses. Parameters were incorporated into the model. We calculated the AUC of multivariable model and plotted ROC curve (Fig. 2). The AUC of this model was 0.743 , suggesting good fitting. Each variable in nomogram was scored. The cumulative sum of these scores was the "total score" that predicted the probability of complication (Fig. 3).

\section{Discussion}

The logistic regression model is an efficient tool to evaluate the incidence of PCC in patients with NSCLC under VATS. In this study, we observed that the incidence of PCCS after VATS was generally higher than $10 \%$, and the incidence of PCCs was low. The model has a strong ability to predict the prognosis of NSCLC, with a positive predictive value of 0.743 . In external verification, the sensitivity and specificity were still relatively high, indicating that our risk prediction model had a good performance in predicting PCCs in patients after NSCLC.

The preoperative changes in ALB, ALB and PNI affect the incidence of PCC after endoscopic surgery in lung cancer patients. Many studies have found that preoperative serum albumin can be used as an important indicator for predicting postoperative results and managing comorbidities [8, 11, 12, 28-31]. 
Malnutrition is a risk factor that can be optimized before surgery, and an accurate predictor of adverse reactions after surgery. A prospective cohort study exploring postoperative complications of major abdominal surgery found that $\triangle A L B \geq 10 \mathrm{~g} / \mathrm{L}$ produced a sensitivity of $77.1 \%$ and a specificity of $67.2 \%$ (AUC: $78.3 \%$ ) to predict complications. Patients with $\triangle \mathrm{Alb} \geq 10 \mathrm{~g} / \mathrm{L}$ on POD 1 had a three-fold increase in the overall risk of postoperative complications [11]. Another prediction model for short-term postoperative complications of gastric cancer showed that with a cut-off $\triangle A L B$ of $19.0 \%$, patients with $\triangle A L B \geq 19.0 \%$ had a higher risk of postoperative complications ( 62.3 vs. $13.7 \%, P<0.001$ ), and longer postoperative hospital stay (22.1 13.5 vs. $17.54 .2, \mathrm{P}<0.001)$ than those with $\triangle \mathrm{ALB}<19.0 \%$ [8]. Another prediction model for postoperative complications of lung cancer also reached the same conclusion[31]. Not only abnormal serum albumin before and after surgery, preoperative hypoalbuminemia is also a strong risk factor for PCCs. When the serum ALB was lower than $3.0 \mathrm{~g} / \mathrm{L}$, the incidences of almost all complex complications showed an upward trend [29]. However, some researchers have proposed that ALB cannot be used as an independent biomarker of malnutrition. These visceral proteins can only be used as a marker of nutritional status among patients in a stable state. Therefore, studies have begun to use the prognostic nutritional index (PNI) as an immunonutrient index to predict PCCs of various types of malignant tumors. Other studies found that low preoperative PNI was an important independent predictor of Clavie-Dindo CII PCCs [13]. In studies related to lung transplantation, multiple regression analysis showed that even after adjusting for other confounding factors, the preoperative PNI score was significantly correlated with postoperative survival, which indicated that PNI can be used as a parameter for evaluating the prognosis of lung transplantation [32]. Age and $\mathrm{CCl}$ are sensitive indicators of the incidence of PCCs after endoscopic surgery in lung cancer patients[3].

This study found that advanced age was also associated with postoperative complications, which is consistent with many other studies. A study of Kim revealed that $>60$ years old was a high-risk factor for postoperative infection, bringing with a risk 1.45 times of that in the low-age group. In prospective studies of lung transplantation, age was also an independent prognostic factor of PCCs [32]. However, more studies suggested that frailty could better predict postoperative outcomes than age. Among the 19 articles reviewed by Beggs, eight showed that frailty was significantly associated with mortality and PCCs [33]. As more and more patients over 75 years of age receive VATS, the vulnerability may have considerable value in evaluating this tool for perioperative evaluation. A short-term prognostic model for the outcome of hepatobiliary, pancreatic, spleen and bile duct surgery showed that the risk index of physical weakness (RAI) was a quick predictor of comorbidities and performance status [34]. Another prospective study clearly concluded that the frailty measurement was better than the stratification by age, and the Rockwood vulnerability index could indicate the outcome of elderly patients receiving EGS. On the contrary, age and American anesthesiologist score (ASA) cannot predict postoperative and major complications (ASA in Table 2) [16].

$\mathrm{CCl}$ is also an indicator for evaluating preoperative frailty, and predict complications after surgery. In a study of Whitmore, although neither CCI nor ASA scores could completely predict the occurrence of PCCs, $\mathrm{CCl}$ was beneficial to consult and evaluate patients with spinal surgery before surgery [35] 
Studies have also compared the diagnostic abilities of (Chalson Comorbidity Score, Elixhauser Comorbidity Score, Comorbidity Score, Centers for Medicare and Medicaid Services-Graded Disease Category [CMS-HCC]) with that of prescription-based chronic disease scores (CDS), finding that almost all scores were better than prescription-based scores, and combining the two scores does not improve the predictive value [36]. More CCl-related studies have suggested using age-adjusted $\mathrm{CCl}$ for predicting postoperative tumor complications [37-39]. Tomonari A also found that higher $\mathrm{CACl}$ was an independent predictor of poor survival in a retrospective study of pancreatic cancer. In this study, age and $\mathrm{CCl}$ showed a correlation with complications in both univariate and multivariate analyses. In order to avoid interference between the two factors during modeling, $\mathrm{CCI}$ replaced $\mathrm{CACl}$.

Intraoperative conversion to thoracotomy and LSHS were related to the incidence of PCC in lung cancer patients after endoscopic surgery. A conclusion is drawn from the analysis of the causes of accidental thoracotomy during thoracoscopic lobectomy for lung cancer. Respiratory complications were more common in the conversion group $(p=0.012)$, but accidental thoracotomy did not increase the overall surgical morbidity and mortality [40]. A multi-center retrospective analysis in Italy showed that unplanned thoracotomy was significantly associated with postoperative morbidity [15]. Among Bertolaccini L's research, intraoperative vascular injury, pleural adhesions, age $>60$ years and resection locations were all factors that affect the incidence of PCCs and mortality. Experience was an independent factor to predict conversion [15]. However, studies of the same type put forward different points of view. A single-center study concluded that there was no significant difference in survival and recurrence between groups with successful VATS and conversion to thoracotomy, but the operation time and hospital stay were longer in the group with conversion to thoracotomy [14]. Another retrospective study also showed that unplanned thoracotomy did not affect the prognosis. [41]

In this study, we found that patients with a long history of smoking before surgery had a significantly higher incidence of PCCs than patients who had never smoked. A retrospective study in Japan found that smokers had a higher PCCs incidence than non-smokers. The incidence of respiratory-related complications gradually increased with the number of smoking years [42]. Another retrospective study of 666 patients in Jampan found that long-term smoking history before surgery and TNM stage were predictors of complications after pneumonectomy [25]. However, Matsuoka K believed that the length of time to quit smoking before surgery had no relationship with the frequency of PCCs, even if smoking restarted within two weeks before surgery. Related studies also found that LSHS before surgery was more correlated with postoperative complications of foot and ankle surgery, adult spinal deformity surgery, and head and neck microvascular reconstruction surgery than those who had never smoked $[23,24,43]$.

There are still some limitations in this study. First, this study is limited by the inherent weak points of single center retrospective analysis, and the potential selection bias may weaken the predictive ability of our prediction model, because PCCs arises from a series of complex factors. In addition, the sample size of a single center may limit the reliability of this analysis. Second, although the predictive power of frailty index is higher than that of age, it is impossible to collect relevant indexes to calculate frailty index prospectively because this study is a retrospective analysis. Third, the accuracy of incidence calculation 
is likely to depend on the clinical experience of surgeons. However, quantitative analysis of human experience may be difficult.

In conclusion, age, $\mathrm{PNI}, \mathrm{CCl}, \mathrm{ALB}$ and conversion to thoracotomy have significant correlation with thoracoscopic surgery and PCCs in patients with non-small cell lung cancer. Therefore, preoperative nutritional status, age, complications and other factors should be comprehensively evaluated to maximize surgical benefits. A larger prospective analysis should be performed in the future to verify our results.

\section{Declarations}

\section{Acknowledgements}

Thanks to the Professor Zhihang Peng, Department of Epidemiology and Biostatistics, School of Public Health, Nanjing Medical University for guidance. Thanks to all the participants for their help.

\section{Authors' contributions}

WK: conceptualization, data collection, investigation. XDR, YY: writing

original draft, software, analysis. WY: conceptualization, analysis. SSB, ZMJ:

methodology. WSS: resources. LW: formal analysis. PZH: writing-original draft, modification. WJQ: supervision, project administration. All authors have read and approved the final manuscript.

\section{Funding}

This work was supported in part by the Natural Science Foundation of China (81871115), Primary Research and Developement Plan of Jiangsu Province (BE2018747), Jiangsu Provincial Key Discipline of Medicine (ZDXKA2016003), National Key R\&D Program of China (NO.2018YFC2002100, 2018YFC2002102). The grant funders had no role in the study design, data collection, analysis and interpretation, or preparation of the manuscript.

\section{Availability of data and materials}

The datasets used and/or analyzed during the current study are available from the corresponding author on reasonable request.

\section{Ethics approval and consent to participate}

The work was approved by the ethical committee of Nanjing Medical University ("F","CH","Nanjing Med U", "FWA00001501", "NANJING", 11/21/2004), and an IRB (Institutional Review Board) approval was given prior to this study. I have read and have abided by the statement of ethical standards for manuscripts submitted to BMC Pulmonary medicine. All methods were carried out in accordance with relevant 
guidelines and regulations. The need for informed consent was waived by the ethical committee of Nanjing Medical University ("F","CH","Nanjing Med U", "FWA00001501", "NANJING", 11/21/2004) due to its retrospective nature.

\section{Consent for publication}

Not applicable.

\section{Competing interests}

The authors declare that they have no competing interests.

\section{References}

1. Zhou M, Wang $H$, Zeng $X$, et al. Mortality, morbidity, and risk factors in China and its provinces, 1990-2017: a systematic analysis for the Global Burden of Disease Study 2017, Lancet, 2019, 28; 394(10204): 1145-58.

2. Postmus $P E$, Kerr KM, Oudkerk M, et al. Early and locally advanced non-small-cell lung cancer (NSCLC): ESMO Clinical Practice Guidelines for diagnosis, treatment and follow-up, Ann Oncol, 2017, 1(28), 1-21.

3. Kent $\mathrm{M}$, Wang $\mathrm{T}$, Whyte $\mathrm{R}$, et al. Open, video-assisted thoracic surgery, and robotic lobectomy: review of a national database, Ann Thorac Surg, 2014, 97(1):236-42.

4. Jung HS, Kim HR, Choi SH, et al. Clinical feasibility and efficacy of video-assisted thoracic surgery (VATS) anatomical resection in patients with central lung cancer: a comparison with thoracotomy, J Thorac Dis, 2015, 7(10):1774-9.

5. Laursen L, Petersen RH, Hansen HJ, et al. Video-assisted thoracoscopic surgery lobectomy for lung cancer is associated with a lower 30-day morbidity compared with lobectomy by thoracotomy, Eur $\mathrm{J}$ Cardiothorac Surg, 2016, 49(3): 870-5.

6. Solaini L, Prusciano F, Bagioni P, et al. Video-assisted thoracic surgery (VATS) of the lung: analysis of intraoperative and postoperative complications over 15 years and review of the literature, Surg Endosc, 2008, 22(2):298-310.

7. Smetana GW, Lawrence VA, Cornell JE, et al. Preoperative pulmonary risk stratification for noncardiothoracic surgery: Systematic review for the American College of Physicians, Ann Intern Med, 2006, 144: 581-95,

8. Ai S, Sun F, Liu Z, et al. Change in serum albumin level predicts short-term complications in patients with normal preoperative serum albumin after gastrectomy of gastric cancer, ANZ J Surg, 2019, 89(7): 297-301.

9. Shigeishi H, Ohta K, Takechi M, et al. Risk factors for postoperative complications following oral surgery, J Appl Oral Sci, 2015, 23(4): 419-23. 
10. Ge X, Cao Y, Wang H, et al. Diagnostic accuracy of the postoperative ratio of C-reactive protein to albumin for complications after colorectal surgery, World J Surg Oncol, 2017, 15(1): 15.

11. Labgaa I, Joliat GR, Kefleyesus A, et al. Is postoperative decrease of serum albumin an early predictor of complications after major abdominal surgery? A prospective cohort study in a European centre, BMJ Open, 2017, 7(4): e013966.

12. Loftus TJ, Brown MP, Slish JH, et al. Serum Levels of Prealbumin and Albumin for Preoperative Risk Stratification, Nutr Clin Pract, 2019, 34(3): 340-8.

13. Okada S, Shimada J, Teramukai S, et al. Risk Stratification According to the Prognostic Nutritional Index for Predicting Postoperative Complications After Lung Cancer Surgery, Ann Surg Oncol, 2018, 25(5): 1254-61.

14. Sezen CB, Bilen S, Kalafat CE, et al. Unexpected conversion to thoracotomy during thoracoscopic lobectomy: a single-center analysis, Gen Thorac Cardiovasc Surg, 2019, 67(11):969-75.

15. Bertolaccini L, Davoli F, Pardolesi A, et al. Conversion due to vascular injury during video-assisted thoracic surgery lobectomy: A multicentre retrospective analysis from the Italian video-assisted thoracic surgery group registry, Eur J Surg Oncol, 2019, 45(5): 857-62.

16. Joseph B, Zangbar B, Pandit V, et al. Emergency General Surgery in the Elderly: Too Old or Too Frail? J Am Coll Surg, 2016, 222(5): 805-13.

17. Ray S, Mehta NN, Mangla V, et al. A Comparison Between the Comprehensive Complication Index and the Clavien-Dindo Grading as a Measure of Postoperative Outcome in Patients Undergoing Gastrointestinal Surgery-A Prospective Study, J Surg Res, 2019, 244:417-24.

18. Park JS, Kim HK, Choi YS, et al. Unplanned conversion to thoracotomy during video-assisted thoracic surgery lobectomy does not compromise the surgical outcome, World J Surg, 2011, 35(3): 590-5.

19. Reichert M, Pösentrup B, Hecker A, et al. Thoracotomy versus video-assisted thoracoscopic surgery (VATS) in stage III empyema-an analysis of 217 consecutive patients, Surg Endosc, 2018, 32(6): 2664-75.

20. Bongiolatti S, Gonfiotti A, Viggiano D, et al. Risk factors and impact of conversion from VATS to open lobectomy: analysis from a national database, Surg Endosc, 2019, 33(12): 3953-62.

21. Bettin CC, Gower K, McCormick K, et al. Cigarette smoking increases complication rate in forefoot surgery, Foot Ankle Int, 2015, 36(5): 488-93.

22. Yousefzadeh A, Chung F, Wong DT, et al. Smoking Cessation: The Role of the Anesthesiologist, Anesth Analg, 2016, 122(5):1311-20.

23. De la Garza Ramos R, Goodwin CR, Qadi M, et al. Impact of Smoking on 30-day Morbidity and Mortality in Adult Spinal Deformity Surgery, Spine, 2017, 42(7): 465-70.

24. Crippen MM, Patel N, Filimonov A, et al. Association of Smoking Tobacco with Complications in Head and Neck Microvascular Reconstructive Surgery, JAMA Facial Plast Surg, 2019, 21(1): 20-6.

25. Fukui M, Suzuki K, Matsunaga T, et al. Importance of Smoking Cessation on Surgical Outcome in Primary Lung Cancer, Ann Thorac Surg, 2019, 107(4):1005-9. 
26. Christopher C, Robert J, Brian E, et al. Incidence, management and outcomes of intraoperative catastrophes during robotic pulmonary resection. Ann Thorac Surg. 2019, 108(5): 1498-504.

27. Steyerberg EW, Vergouwe Y. Towards better clinical prediction models: seven steps for development and an ABCD for validation. Eur Heart J, 2014, 35(29): 1925-31.

28. Gruppo M, Angriman I, Martella $B$, et al. Perioperative albumin ratio is associated with post-operative pancreatic fistula, ANZ J Surg, 2018, 88(7): 602-5.

29. Nelson CL, Kamath AF, Elkassabany NM, et al. The serum albumin threshold for increased perioperative complications after total hip arthroplasty is 3, 0 g/dL, Hip Int. 2019, 29(2): 166-71.

30. Li N, Qiao H, Guo JF, et al. Preoperative hypoalbuminemia was associated with acute kidney injury in high-risk patients following non-cardiac surgery: a retrospective cohort study, BMC Anesthesiol, 2019, 19(1): 171.

31. Li P, Li J, Lai Y, et al. Perioperative changes of serum albumin are a predictor of postoperative pulmonary complications in lung cancer patients: a retrospective cohort study, J Thorac Dis, 2018, 10(10): 5755-63.

32. Kim CY, Kim SY, Song JH, et al. Usefulness of the preoperative prognostic nutritional index score as a predictor of the outcomes of lung transplantation: A single-institution experience, Clin Nutr, 2019, 38(5): 2423-9.

33. Mclsaac DI, MacDonald DB, Aucoin SD, et al. Frailty for Perioperative Clinicians: A Narrative Review, Anesth Analg, 2020, 130(6):1450-60.

34. Windt DJ, Bou-Samra P, Dadashzadeh ER, et al. Preoperative risk analysis index for frailty predicts short-term outcomes after hepatopancreatobiliary surgery, HPB, 2018, 20(12):1181-8.

35. Whitmore RG, Stephen JH, Vernick C, et al. ASA grade and Charlson Comorbidity Index of spinal surgery patients: correlation with complications and societal costs, Spine J, 2014, 14(1): 31-8.

36. Mehta HB, Dimou F, Adhikari D, et al. Comparison of Comorbidity Scores in Predicting Surgical Outcomes, Med Care, 2016, 54(2):180-7.

37. Ondeck NT, Bohl DD, Bovonratwet $P$, et al. Discriminative ability of commonly used indices to predict adverse outcomes after poster lumbar fusion: a comparison of demographics, ASA, the modified Charlson Comorbidity Index, and the modified Frailty Index, Spine J, 2018(1): 44-52.

38. Asano T, Yamada S, Fujii T, et al. The Charlson age comorbidity index predicts prognosis in patients with resected pancreatic cancer, Int J Surg, 2017, 39:169-75.

39. Nakajo K, Abe S, Oda I, et al. Impact of the Charlson Comorbidity Index on the treatment strategy and survival in elderly patients after non-curative endoscopic submucosal dissection for esophageal squamous cell carcinoma: a multicenter retrospective study, J Gastroenterol, 2019, 54(10): 871-80.

40. Byun CS, Lee S, Kim DJ, et al. Analysis of Unexpected Conversion to Thoracotomy During Thoracoscopic Lobectomy in Lung Cancer, Ann Thorac Surg, 2015, 100(3): 968-73.

41. Park JS, Kim HK, Choi YS, et al. Unplanned conversion to thoracotomy during video-assisted thoracic surgery lobectomy does not compromise the surgical outcome. World J Surg. 2011, 35(3): 590-5. 
42. Fukui M, Suzuki K, Matsunaga T, et al. Importance of Smoking Cessation on Surgical Outcome in Primary Lung Cancer, Ann Thorac Surg, 2019, 107(4): 1005-9.

43. Beahrs TR, Reagan J, Bettin CC, et al. Smoking Effects in Foot and Ankle Surgery: An Evidence-Based Review, Foot Ankle Int, 2019, 40(10): 1226-32.

\section{Figures}
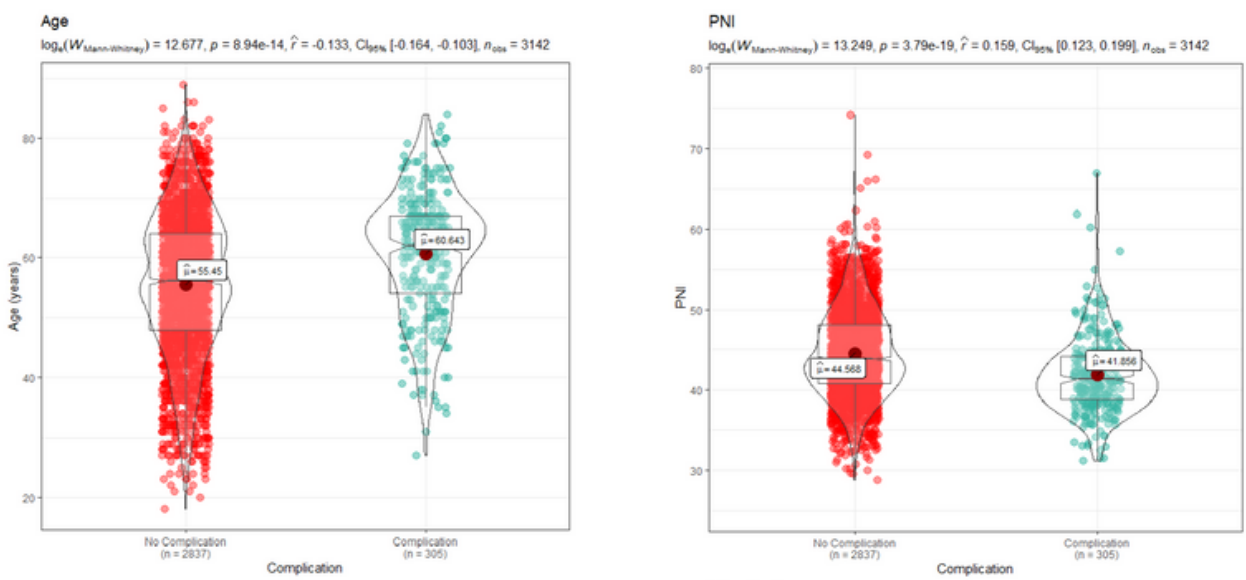

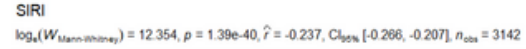
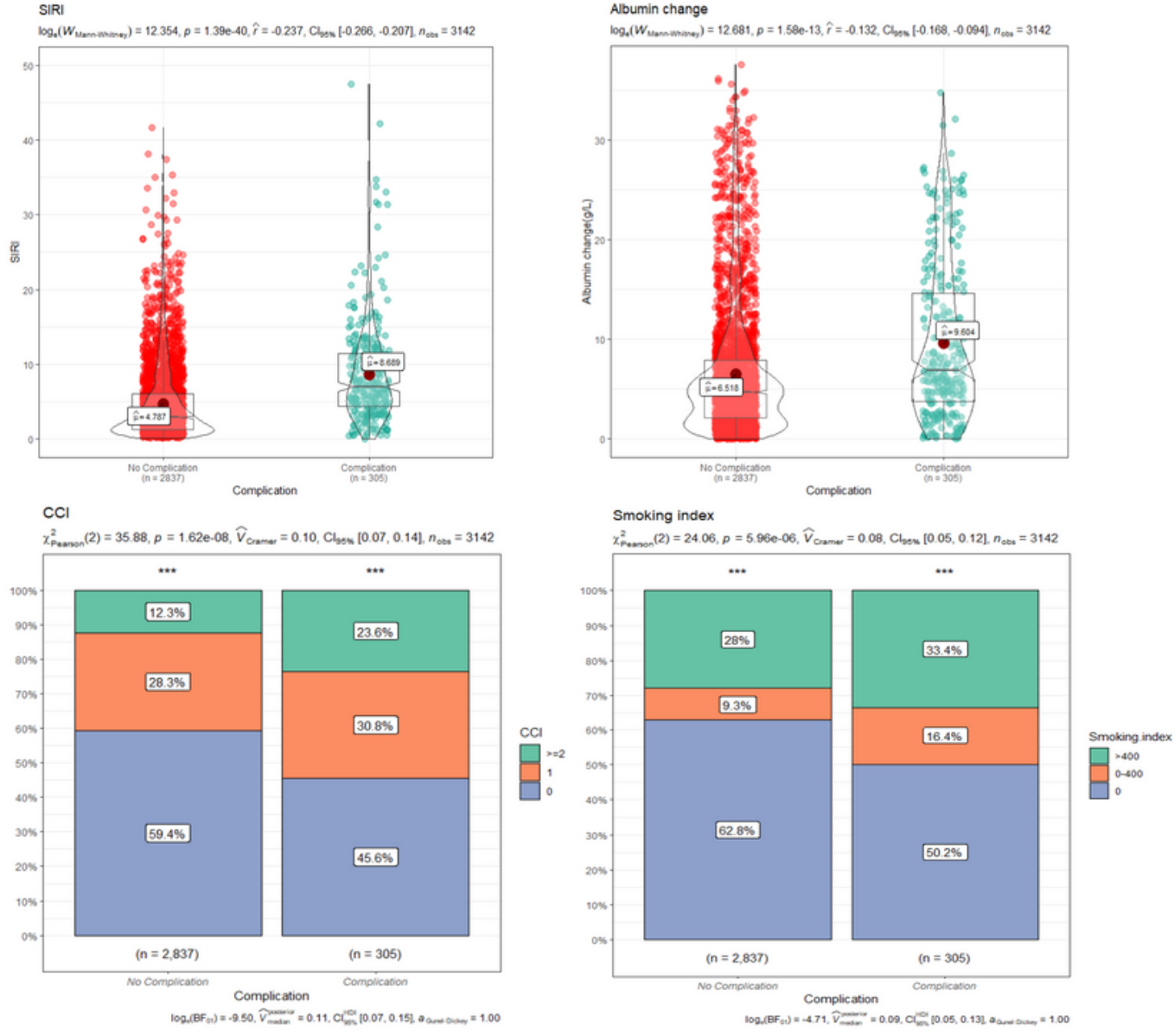


\section{Figure 1}

Age (A); PNI(B); SIRI(C); Albumin change (D); $\mathrm{CCI}(\mathrm{E})$; Smoking index(F) in patients with and without PCCs in patients with VATS.

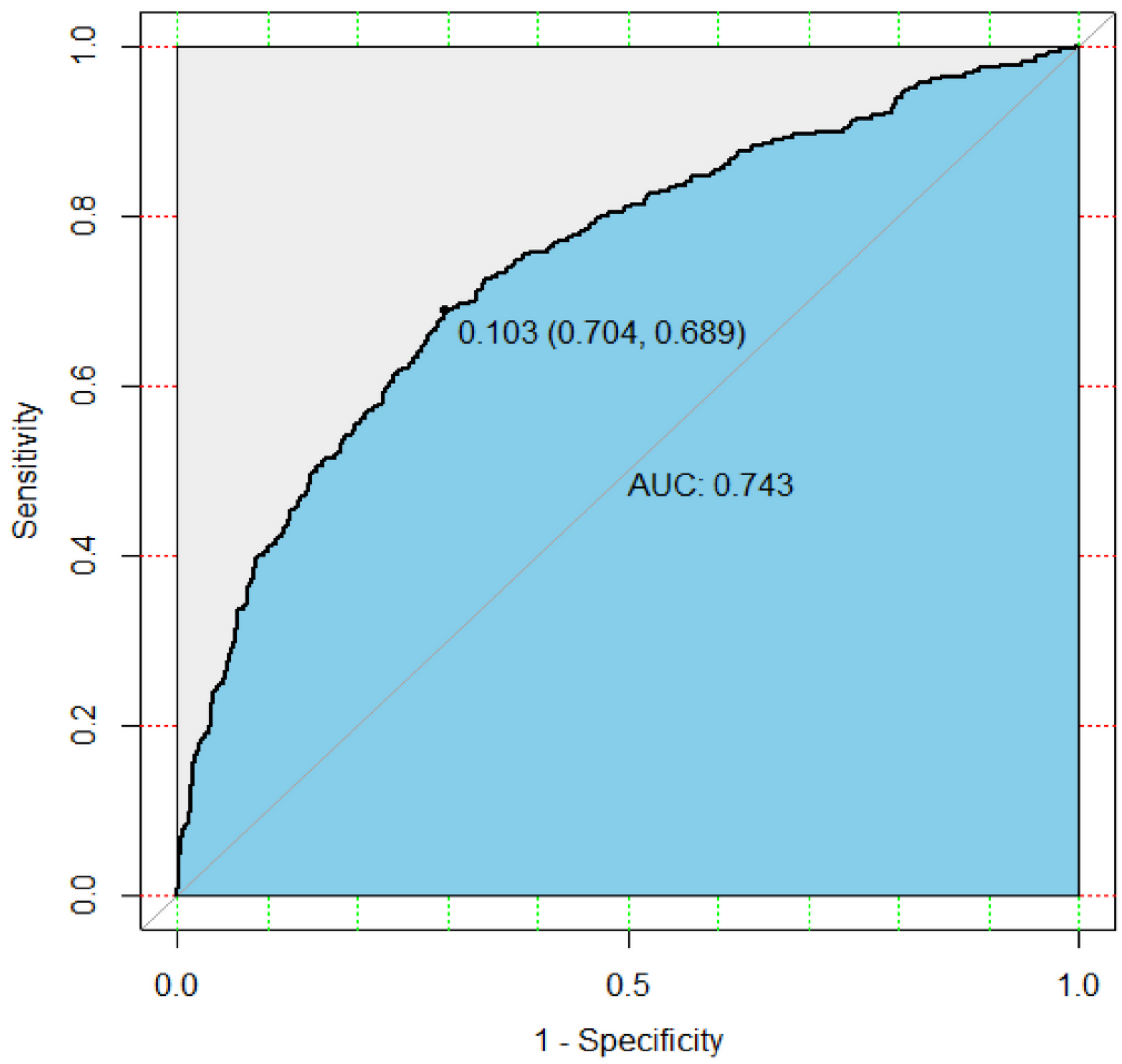

Figure 2

Receiver operating characteristic (ROC) curve to determine the optimal cut-off of multivariate analysis results, showed an AUC of 0.743 . 
Points

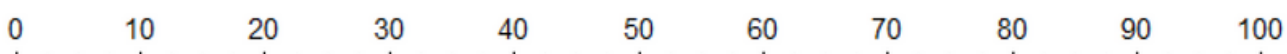

$\mathrm{CCl}$

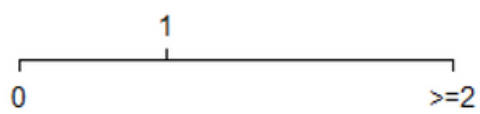

Age

\begin{tabular}{rrrrrrrrr}
\hline 10 & 20 & 30 & 40 & 50 & 60 & 70 & 80 & 90
\end{tabular}

PNI

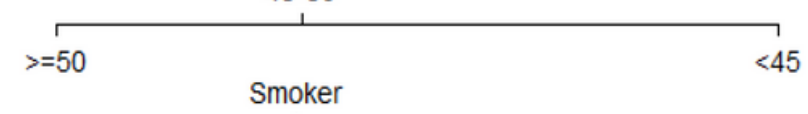

Smoking

$$
\text { No-smoker }
$$

Conversion to thoracotomy

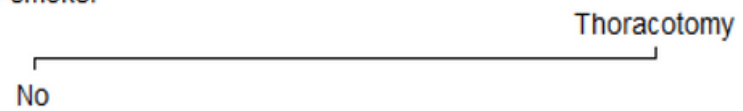

$\triangle \mathrm{ALB}$

\begin{tabular}{lllllllll}
\hline 0 & 5 & 1 & 1 & 1 & 1 & 1 & \\
\hline
\end{tabular}

Total Points

\begin{tabular}{lllllllllllllll}
\hline & 20 & 40 & 60 & 80 & 100 & 120 & 140 & 160 & 180 & 200 & 220 & 240 & 260 & 280
\end{tabular}

Risk

\begin{tabular}{lllllll}
\hline 0.1 & 0.2 & 0.3 & 0.4 & 0.5 & 0.6 & 0.7
\end{tabular}

Figure 3

Nomogram for predicting the risk of PCCs. 